\title{
Cognitive Radio Modulation Techniques
}

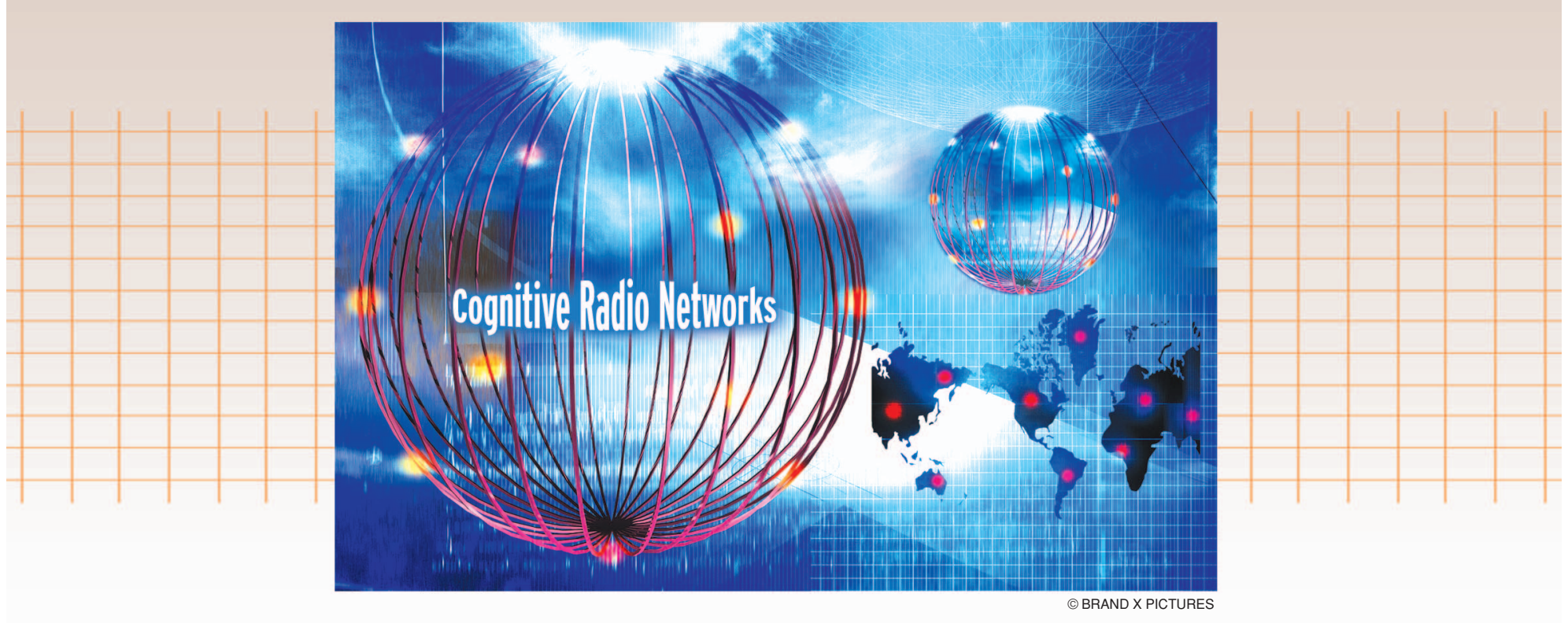

\section{Reducing mutual interference}

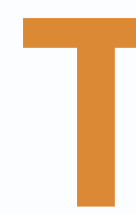

he growing demand on wireless communication systems to provide high data rates has brought with it the need for a flexible and efficient use of the spectrum resource, which is a scarce commodity. The regional spectrum allocation policy counteracts the free mobility of radio communication equipment. The vast majority of the available spectral resources have already been licensed, so it appears that there is little or no room to add any new services, unless some of the existing licenses are discontinued. Furthermore, recent studies and measurements have shown that vast portions of the licensed spectra are rarely used due to the inflexible spectrum regulations. The whole idea behind cognitive radio (CR) use is that it

Digital Object Identifier 10.1109/MSP.2008.929365 should prompt effective spectrum use, since intelligence and learning processes aid the radio system to access the spectrum effectively. The CR system has learning and understanding capabilities so that the stated goals may be achieved. In this article, we shall limit the scope of cognition to reduce mutual interference between CR-based rental (unlicensed) users (RUs) and licensed users (LUs) and in providing coexistence between them. It is expected that the rental users will be allowed to transmit and receive data over portions of spectra when primary (i.e., licensed) users are inactive. In this article, we will introduce the modulation strategies employed to realize a coexistence between the CR-based rental system and the licensed system. This is done in such a way that the RUs are invisible to the LUs. We consider the rental user accesses the unoccupied LU band in overlay fashion. 


\section{INTRODUCTION}

$\mathrm{CR}$, as defined in [1], is an intelligent wireless communication system that is aware of its surrounding environment and uses that understanding by building to learn from the environment and adapt its internal states to variations in incoming radio frequency (RF) stimuli by changing its operating parameters in such a way that highly reliable communication and efficient utilization of the radio system can be achieved. The key words in this definition are awareness, intelligence, learning, adaptivity, reliability, and efficiency. Mitola in [2] developed the notion that CR signifies a radio that employs model-based reasoning to achieve a specified level of competence in radio related domains. This includes supervised and unsupervised learning, and it is assumed to be implemented by software radio or software defined radio architecture.

In [2], spectrum pooling is addressed as a mechanism where the current spectrum owners allow portions of their spectrum to be utilized by RUs which apply the CR system in their transmission for certain periods. This mechanism is applied to consider the actual spectrum occupancy condition where the allocated spectrum is not occupied most of the time. In fact, some portions of the spectrum are rarely used [3]. The spectrum owners, who are known as the LUs, are ordinary mobile terminals and their associated base stations. They do not possess much intelligence. The RUs, on the other hand, should possess the intelligence to sense the spectrum and use whatever resources are available when they need them. At the same time, the RUs should renounce the spectrum when an LU begins transmission. The RU access should be done in such a way that the neighboring LUs will not be aware of its existence. This can be accomplished by assuring that the neighboring LUs will not be interrupted or interfered with as the RU accesses the spectrum hole adjacent to the LU's band. This stipulation can be technically interpreted as in overlay opportunistic RU (CR) access mode where part of the RU spectrum which is located on the neighboring occupied LU band should be shaped to be low as possible so that it will not significantly interrupt the LU transmission as well as reducing mutual interference between the RU and LU, hence reliable communications can be accomplished. This shaping can be interpreted as a sidelobe suppression technique. In this article, the techniques used to fulfill the coexistence between LU and RU in opportunistic spectrum access and to also achieve the CR objectives are described. We shall review the multicarrier and single carrier approach using either the Fourier or wavelet basis function as the CR modulation technique.

\section{MULTICARRIER CR SYSTEM}

Orthogonal frequency division multiplexing (OFDM) is well known as a spectrally efficient modulation mode due to its overlapped carrier spectrum which still remain orthogonal to each other, and it gives a higher bit rate compared to the single carrier transmission. Recently research interest has been developed in replacing the static sines/cosines basis function in OFDM with wavelet bases due to its flexibility and adaptability which can be tailored to satisfy the engineering demand. The next subsections will describe how these two multicarrier modulation techniques can be applied in cognitive radio systems.

\section{OFDM-BASED CR SYSTEM}

OFDM has recently been introduced as a strong multicarrier modulation scheme candidate to be applied in CR [4], [5]. The rationale is that any $\mathrm{CR}$ system needs to sense the spectrum and that involves some sort of spectral analysis. Fast Fourier transform (FFT) can be used for spectral analysis while at the same time acting as an OFDM demodulator. It has therefore been suggested that OFDM should be seen as the proper candidate for multicarrier-based CR systems. OFDM also has the capability to notch the parts of its carriers which are coincidentally within the region of the LU's band. Such OFDM flexibility simplifies the application of $\mathrm{CR}$ dynamic spectrum access. The OFDM carriers notching technique is introduced in [4] and [5] and it is called spectrum pooling. The shortcomings of the OFDM root back to the large side lobes of the frequency response of filters that characterize the channel associated with each subcarrier. The large sidelobes result in significant interference between the subcarriers originating from different RUs and between LUs and RUs.

The sidelobes of the Fourier-based OFDM can be observed from its power spectral density (PSD),

$$
\operatorname{PSD}(f)=\frac{1}{N_{\mathrm{FFT}}}\left|\sum_{m=0}^{N_{\mathrm{FFT}-1}} \sqrt{p_{m}} S_{m} \int_{-(1+\alpha) \frac{T_{u}}{2}}^{(1+\alpha) \frac{T_{u}}{2}} g(t) e^{-j 2 \pi\left(f-f_{m}\right)^{t}} d t\right|^{2}
$$

where $N_{\text {FFT }}$ is the number of FFT points, $T_{u}$ is the useful signal duration, $g(t)$ is the window function, and $\alpha$ is the roll off factor of the window, while $f_{m}, p_{m}$, and $S_{m}$ are the respective frequency, allocated power and the symbol from quadrature amplitude modulation (QAM) or phase shift keying (PSK) mapping on the subcarrier $m$. It is shown in (1) that parameters such as allocated power $\left(p_{m}\right)$, symbol amplitude $\left(S_{m}\right)$ and window $(g(t))$ can be set to resolve the sidelobes problem.

\section{SIDELOBE SUPPRESSION BY WINDOWING AND CARRIERS DEACTIVATION}

In [4], the extension of each OFDM block with long cyclic prefix and suffix samples and the application of some windowing to reduce the sidelobes of the subcarrier channels is proposed. Obviously, this solution occurs at the expense of bandwidth loss because excessive time has to be allocated to cyclic extensions which otherwise could be used for data transmission. Furthermore in [4], the interference reduction method is extended by deactivating subcarriers located adjacent to the LU's band which provides a kind of shield to the LUs. One commonly used window is the raised cosine window [4]. The other available forms are Bartlett [6], better than raised cosine (BTRC) [7], and flipped inverse hyperbolic secant (farchsech) [8] windows. Of these windows, it is the farchsech window that provides the 


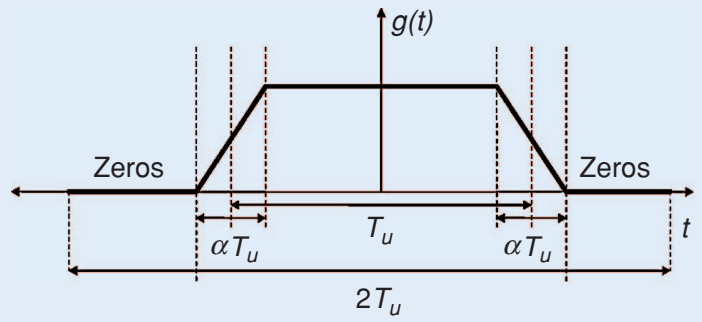

(a)

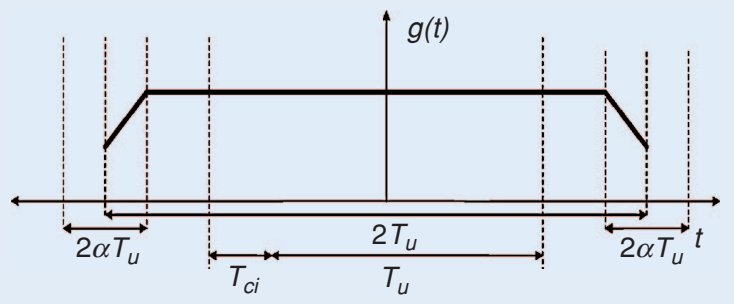

(b)

[FIG1] (a) A window design that influences the transmitted signal and (b) a window design that does not influence the transmitted signal [11].

lowest sidelobes. The window is designed according to (2), shown at the bottom of the page, where $\gamma=2 \ln (\sqrt{3}+2) /\left(\alpha T_{u}\right), \alpha$ is the roll-off factor, and $T_{u}$ is the useful OFDM symbol interval (without guard interval). The shape of the window in the region of $T_{u}(1+\alpha) / 2 \leq|t| \leq T_{u}(1+\alpha) / 2$ determines how rapidly the OFDM spectrum falls to zero. The PSD will hit zero on the frequencies in the interval of $1 / T_{u}$.

According to [9], the duration of the OFDM signal should be $2 T_{u}$ in order to complete a total $2 N_{\text {FFT }}$ samples to preserve the orthogonality, and zeros are added in the region outside the window $g(t)$. If the duration of the OFDM signal is only $T_{u}(1+\alpha)$ then the window will affect the transmitted signal, and as a consequence an error floor will be formed. Figure 1(a) shows an example of a Bartlett window with an OFDM signal $T_{u}(1+\alpha)$ duration which affects the transmitted signal. In an effort to avoid the error floor, the applied window must not influence the signal during its effective period [10]. To fulfill this requirement the window forms are expanded, so that new $T_{u}$ becomes $2 T_{u}$, and $\alpha$ is restricted in the range of $0 \leq \alpha \leq\left(1-T_{G I} / T_{u}\right) / 2$, where $T_{G I}$ is the guard interval duration. The orthogonality is preserved since the PSD hits zeros in the $1 /\left(2 T_{u}\right)$ interval at the expense of the longer duration of the OFDM signal $\left(2 T_{u}(\alpha+1)\right)$. The solution is that the window is truncated to fit the $2 T_{u}$ OFDM duration, as depicted in Figure 1(b) [11]. The orthogonality is preserved by applying the rectangular receiver filter with $T_{u}$ duration, that is implemented by DFT [10]. In this way there is more freedom to shape the window in the $T_{u}(1+\alpha) \leq|t| \leq T_{u}$ region as long as its PSD on the symbol boundaries remains low, e.g., Gaussian window or half sine window [12].

By referring to (1), the deactivating carriers adjacent to the licensed band will provide flexible guard bands that will make the PSD sidelobes of the RUs' OFDM signal on the licensed band lower. In OFDM, bits errors are typically concentrated in a set of severely faded subcarriers. This is due to the channel propagation condition. For this reason, it is possible that the quality of service may not be maintained. Meanwhile, the carriers resource is limited due to the deactivation of the RU carriers. Furthermore, the long OFDM symbol duration due to the windowing reduces the OFDM transmission bit rate. Having adaptive OFDM in CR is thus a solution. The heavily faded and noisy subcarriers are excluded from transmission to improve the overall bit error rate (BER) of the system, and the loss of throughput is counteracted by applying higher order modulation modes to the subcarriers which have better signal to noise ratios (SNR) or BER. The combination of adaptive bit loading and spectrum pooling has been evaluated in [11]. The window was designed in accordance with Figure 1(b). The results show how adaptive bit loading algorithms can outperform the ordinary OFDM.

Recently, (in [13]) the overlapped OFDM symbol transmission with long symbol duration (known as offset OFDM) has been introduced to counteract the throughput loss. The scheme is described in Figure 2. The delay between one OFDM symbol and another is designed in such a way that no intersymbol interference occurs, which means that the next OFDM symbol should start after the end of the useful data part of the previous OFDM symbol. If zeros are inserted at the prefix and suffix points [outside the $T_{G I}$ and $T_{U}$ area as depicted in Figure 1(b)], the useful data of the next OFDM symbol should start after the last useful data of the previous OFDM symbol.

\section{SIDELOBE SUPPRESSION BY CANCELLATION CARRIERS INSERTION}

In [14], instead of having deactivated carriers adjacent to the LU band, these carriers are used to cancel out the sidelobes on the LU band. Figure 3 provides a simple example of how the cancellation carriers work. The spectrum is derived from

$g(t)=\left\{\begin{array}{cc|}\frac{1}{T_{u}}, & 0 \leq|t| \leq \frac{T_{u}(1-\alpha)}{2} \\ \frac{1}{T_{u}}\left[1-\frac{1}{\alpha T_{u} \gamma} \operatorname{arcsech}\left(\frac{1}{\alpha T_{u}}\left(\frac{T_{u}(1+\alpha)}{2}-|t|\right)\right)\right], & \frac{T_{u}(1-\alpha)}{2} \leq|t| \leq \frac{T_{u}}{2} \\ \frac{1}{T_{u}}\left[\frac{1}{\alpha T_{u} \gamma} \operatorname{arcsech}\left(\frac{1}{\alpha T_{u}}\left(|t|-\frac{T_{u}(1-\alpha)}{2}\right)\right)\right], & \frac{T_{u}}{2} \leq|t| \leq \frac{T_{u}(1-\alpha)}{2} \\ 0 & \text { Otherwise }\end{array}\right.$




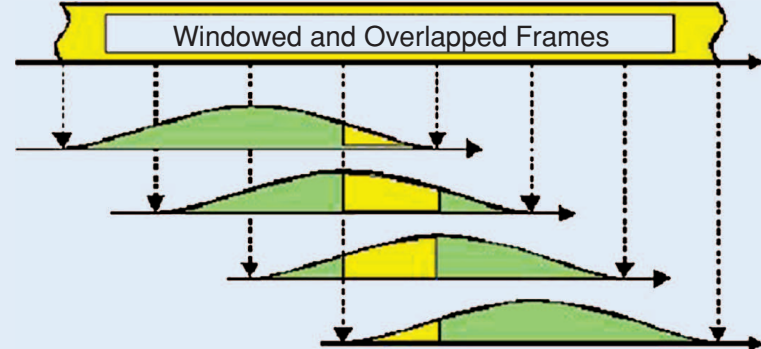

(a)

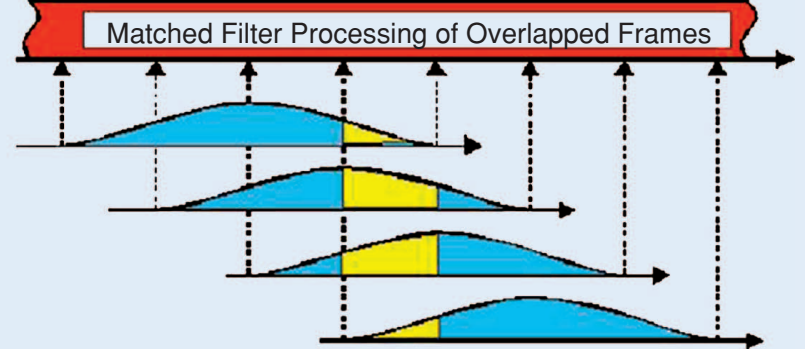

(b)

[FIG2] Overlapped OFDM symbol with zero ISI: (a) reflects the technique applied at the transmitter point while (b) is applied at the receiver point.

nine subcarriers (including the cancellation carriers) and uses a rectangular window which produces a spectrum from the sum of the sinc functions. One cancellation carrier is located on the left edge of the RU band adjacent to the LU band and another one is on the right edge. They are signed by the dark vertical arrows. If we take a look at the OFDM spectrum with carriers deactivation (one carrier on the left and one carrier on the right edge) denoted by the solid curve, we can see that the maximum sidelobe occurs at the frequency point half of carrier spacing from the deactivated carrier which, in Figure 3 is indicated by the coordinate position (X:-4.5,Y:-0.1564). The cancellation carriers can be weighted in such a way that the sum of their spectrums will nullify the OFDM spectrum on that particular coordinate position (e.g., by having a cancellation carrier spectrum coordinate $\left.\left(X_{c 1}=X_{c 2}=-4.5, Y_{c 1}=0.1477, Y_{c 2}=0.0087\right)\right)$. The same technique is also applied for the sidelobes reduction on the right side. The resulting spectrum derived from using the cancellation carriers technique is depicted by the dashed lines. The sidelobe is lower than the sidelobe of the OFDM with the deactivated carriers.

The cancellation carriers can be combined with windowing [14]. Different windows provide different sidelobe levels. Combining a window that gives very low sidelobes (e.g., the farchsech window) with the cancellation carriers technique will lead to a very significant sidelobe (interference) reduction in the LU band.

\section{SIDELOBE SUPPRESSION \\ BY SUBARRIER WEIGHTING}

Subcarrier weighting is introduced in [15]. The weighting can be seen as varying the allocation power $p_{m}$ on each carrier $m$ which, obviously, according to (1) will affect the spec- trum amplitude. By selecting the appropriate weight for each of the carriers the desired low sidelobes can be obtained. The sidelobe reduction by means of carriers weighting is attained at the expense of the BER versus SNR degradation [15], since the weighting will give unequal amounts of transmission power on each of the OFDM carriers. The weighting method was originally used in [16] to counteract the high peak to average power ratio (PAPR) problem in OFDM.

\section{SIDELOBE SUPPRESSION BY MULTIPLE CHOICE SEQUENCE}

In [17] a multiple choice sequence is introduced, where the multiple choice sequence abiding by the symbol constellation approach, the interleaving approach and the phase approach are discussed. The symbol constellation approach is applied by mapping the symbol $S_{m}$ in (1) into $S_{m}^{\prime}(l)$ which is still derived from one of the CS symbols after the QAM or PSK mapping that was previously used to

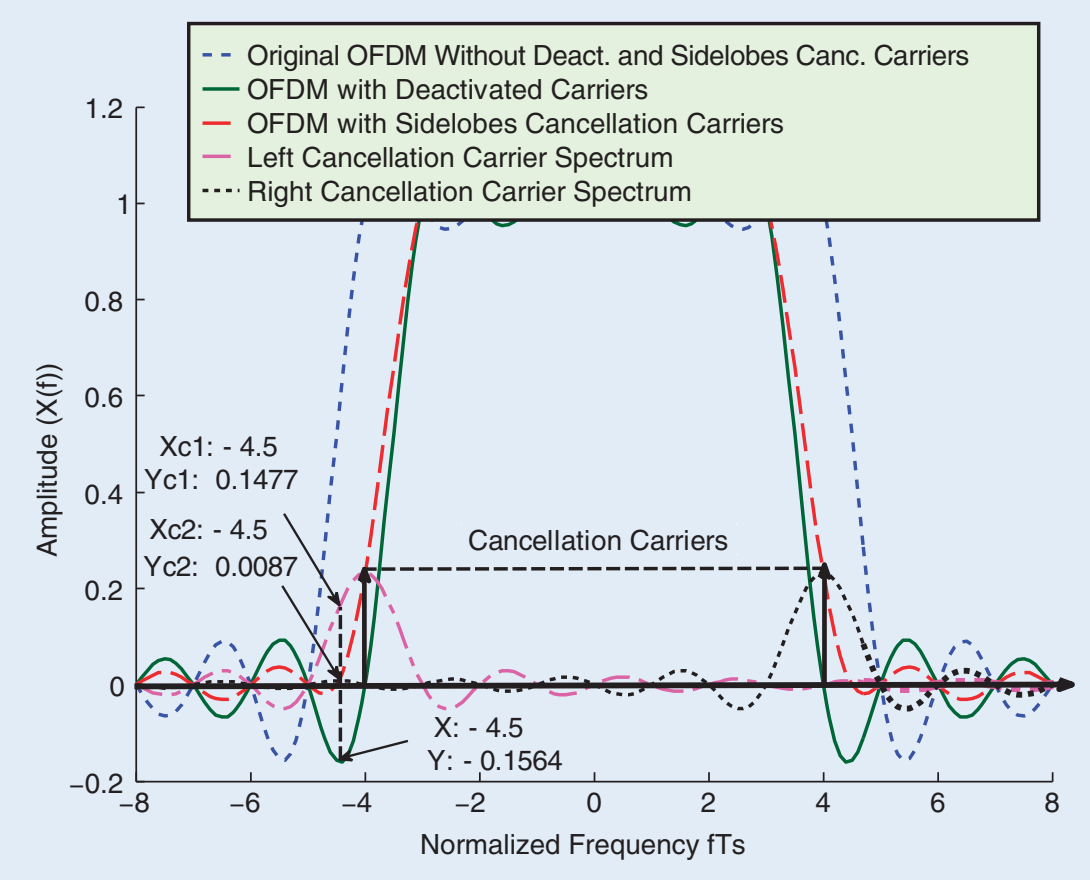

[FIG3] The OFDM spectrum amplitude with the effect of the deactivated carriers or cancellation carriers. 
produce $S_{m}$. CS is the constellation size of the QAM or PSK mapping. The index $l$ ranges from $l=1,2, \ldots, L$, where $L$ is the number of possible alternative values. This means that the symbol $S_{m}$ can be mapped into $L$ possible symbols. By putting the $N_{\text {FFT }}$ symbols of $S_{m}^{\prime}(l)$ into (1), and observing from all $L$ possible PSDs, the one with the lowest sidelobe is then chosen for transmission. An example of simple mapping from $S_{m}$ into $S_{m}^{\prime}(l)$ is given in [18]. The $C S$ points of QAM or PSK are numbered as $0,1, \ldots, C S-1$, hence the symbol $S_{m}$ is assigned to the number $I_{m}\left(I_{m} \in\{0,1, \ldots, C S-1\}\right)$ according to its point position. The $S_{m}^{\prime}(l)$ will be the symbol value on the new number $I_{m}(l)$ that is derived from the addition of the assigned number $I_{m}$ with the random number $D_{m}(l)$ that has $L$ different possible values [17]

$$
I_{m}(l)=I_{m}+D_{m}(l) .
$$

By transmitting the information about the set index information $l$, the receiver can decode the received signal and reconstruct the original $S_{m}$ symbol. By means of the interleaving approach, the $N_{\text {FFT }} S_{m}$ symbols are interleaved by $L$ different interleaver rules,

$$
S^{\prime}(l)=\Pi(l) S
$$

where $\Pi(l)$ is the permutation matrix that will do the interleaving. The matrix $\Pi(l)$ has $L$ different possible matrix values. The permutation matrix $\Pi(l)$ will be available at the transmitter and the receiver. Among $L$ different possible OFDM spectrum using set of symbols $S^{\prime}(l)$, the one with the lowest sidelobes is chosen for transmission. The receiver will use the deinterleaver permutation matrix $\Pi^{1}(l)$ to recover the transmitted data upon receiving the side information index $l$.

In the phase approach, each phase of symbol $S_{m}$ is shifted by random phase $\theta_{n}(l)$ with $L$ different possible values. The phase shifted symbol $S_{m}^{\prime}(l)$ becomes [17]

$$
S_{m}^{\prime}(l)=S_{m} e^{j \theta_{n}(l)}
$$

where $\theta_{n}(l)$ is in the range of between $0-2 \pi$. In the vector notation, the phase shifted signal $S^{\prime}(l)$ is derived from point to point multiplication between the original signal $S$ and the complex number vector derived from $e^{j \theta(l)}$. As in the constellation and interleaver approach, in the phase approach, among $L$ possible sets of vector $\boldsymbol{\theta}(l)$, the one that produces the lowest sidelobes is chosen and applied to the symbol vector $S$. The index information $l$ is transmitted so that the receiver can reconstruct the symbol vector $S$. Apart from the random shift, random amplitude shift can also be applied, but in such cases the BER performance will be affected. Another approach is to only apply the multiple choice sequence technique to the carriers adjacent to the LU band, since these carriers affect the sidelobes magnitude most [17].

\section{SIDELOBE SUPPRESSION BY ADAPTIVE SYMBOL TRANSITION}

The most recent OFDM sidelobes suppression technique is introduced in [18]. Time-domain symbol transition is inserted between two OFDM symbol, and the symbol transition value is obtained in such a way that the signal spectrum or the Fourier transform of the two consecutive time domain OFDM symbol (including the symbol transition) will have low sidelobes. The objective is to minimize the spectrum resulting from two consecutive time domain OFDM symbol without the symbol transition added by the spectrum contributed from the symbol transition on the LU band [18]. The symbol transition will not introduce any intersimbol interference (ISI) but the transmission duration becomes longer, which means that the bit rate is reduced.

Of these methods in suppressing the OFDM sidelobes, the CR system could employ a combination of the introduced techniques (e.g., windowing + cancellation carriers + adaptive bit loading) according to its target service (e.g., higher bit rate, low BER, and considerable complexity) to obtain efficient spectrum access and reliable communications.

\section{WAVELET PACKET-BASED CR SYSTEM}

In multicarrier wavelet packet modulation (MC-WPM), the Fourier transform used in OFDM is replaced by the orthonormal wavelet packet bases. The wavelet packet bases and their dual bases are obtained from perfect reconstruction two-band finiteimpulse response (FIR) filter bank solution using multistage tree-structured paraunitary filter banks derived by cascading two-channel filter banks [19], [20]. The wavelet transform provides higher sidelobes suppression when compared to the rectangular window sidelobes in regular OFDM [21]. In [22], the Daubechies and Haar wavelet-based multicarrier transmission over Rayleigh fading channel were evaluated. Their performances in terms of intersymbol interference (ISI) and intercarrier interference (ICI) have been compared with the Fourier-based OFDM using rectangular window. The wavelet-based multicarrier provides significant ICI power reduction and slight ISI power reduction compared to OFDM.

\section{WAVELET PACKET FILTERBANKS}

The wavelet packet subcarriers (to be used at the transmitter end) are generated through a multichannel filterbank consisting of two cascaded channel filterbanks in which the synthesis filters $\left(H^{\prime}\right.$ and $\left.G^{\prime}\right)$ are applied. The process known as the synthesis procedure consists of binary interpolation (upsampling) by two, filtering and recombination at each level. To demonstrate the generation of the wavelet packet bases process, we shall consider here a cascaded level-three tree structure of the type shown in Figure 4(a). Such an arrangement can give rise 
to eight wavelet packet bases. In general, a level- $J$ tree has to be constructed to generate $M$ bases or subcarrier waveforms. The structure in Figure 4(a) can be simplified by applying the noble identities procedure that will produce multirate systems. Each of the carrier will be upsampled by $M$ and will experience only one tap filtering. The filter is the equivalent filter resulting from the convolution of the combination between low pass and high pass filters according to the branch line of the tree [23]. The high pass filter $H^{\prime}(z)$ with length $L^{\prime}$ can be obtained from the low pass filter $G^{\prime}(z)$ by means of the $G^{\prime}(z)=-z^{-\left(L^{\prime}-1\right)} H^{\prime}\left(-z^{-1}\right)$ equation [24]. The coexistence with the LUs is actualized by shaping its transmission waveform and by adaptively activating or deactivating subcarriers in such a way as to utilize the unoccupied time-frequency gaps of the LU. The idea is to dynamically notch the transmission signal so that it has no or very little time-frequency components competing with the LU. In that way, the CR can seamlessly blend with the LU operation.

The wavelet packet duals (to be used at the receiver end) are also obtained from multichannel filter bank analysis, though the processes are reversed. The duals are obtained from the analysis filters ( $H$ and $G$ ) through the analysis procedure which consists of filtering, decimation (downsampling) by two and decomposition at each stage. Figure 4(b) illustrates the generation of eight wavelet packet duals from a level-three tree cascaded filter bank. The dual filters are derived from paraunitary condition $P(z)+P(-z)=2$, where the product filter $P(z)=H(z) H\left(z^{-1}\right), \quad[24]$ and $H\left(z^{-1}\right)=H^{\prime}(z)$. In the time domain the analysis filters are the complex conjugate time reversed versions of the synthesis filters $\left(h^{\prime}(n)=h^{*}(-n)\right.$ and $\left.g^{\prime}(n)=g^{*}(-n)\right)$.

The wavelet basis function in the application of MC-WPM systems should fulfill some fundamental properties. These properties include the time and band limitation, the orthogonality between the bases and their duals for perfect reconstruction, and the orthogonality between the bases for the unique demodulation of each basis function. Of the available wavelets such as: Coifflets, Daubechies, Haar, and Symlets, the most suitable wavelet that fulfils the required properties is the family of maximum frequency selective filter banks derived from a modified Remez exchange algorithm [24]. It provides a degree of freedom to shape its sidelobes by varying its regularity order, transition bandwidth, and filter length. From the filter with length $L^{\prime}$, the Remez algorithm will produce $L^{\prime}$ equations, $L^{\prime} / 2$ of which will be dedicated for the paraunitary condition, $K$ equations for the regularity order and left $L^{\prime} / 2-K$ for frequency selectivity. The longer the filter length, the greater the degree of freedom will be for the design of the frequency selectivity and the regularity order of the filters. The

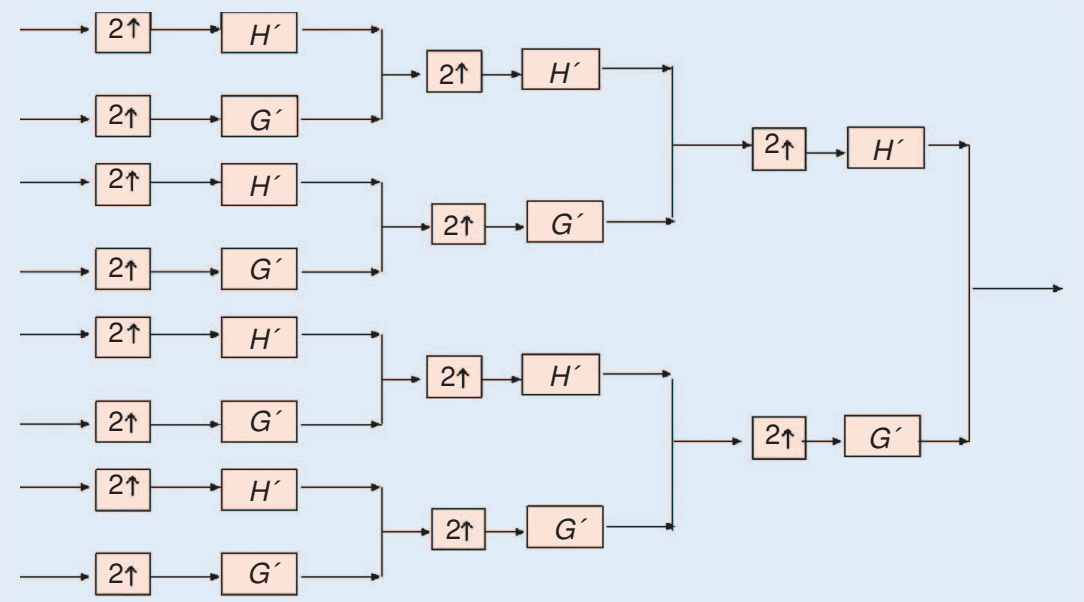

(a)

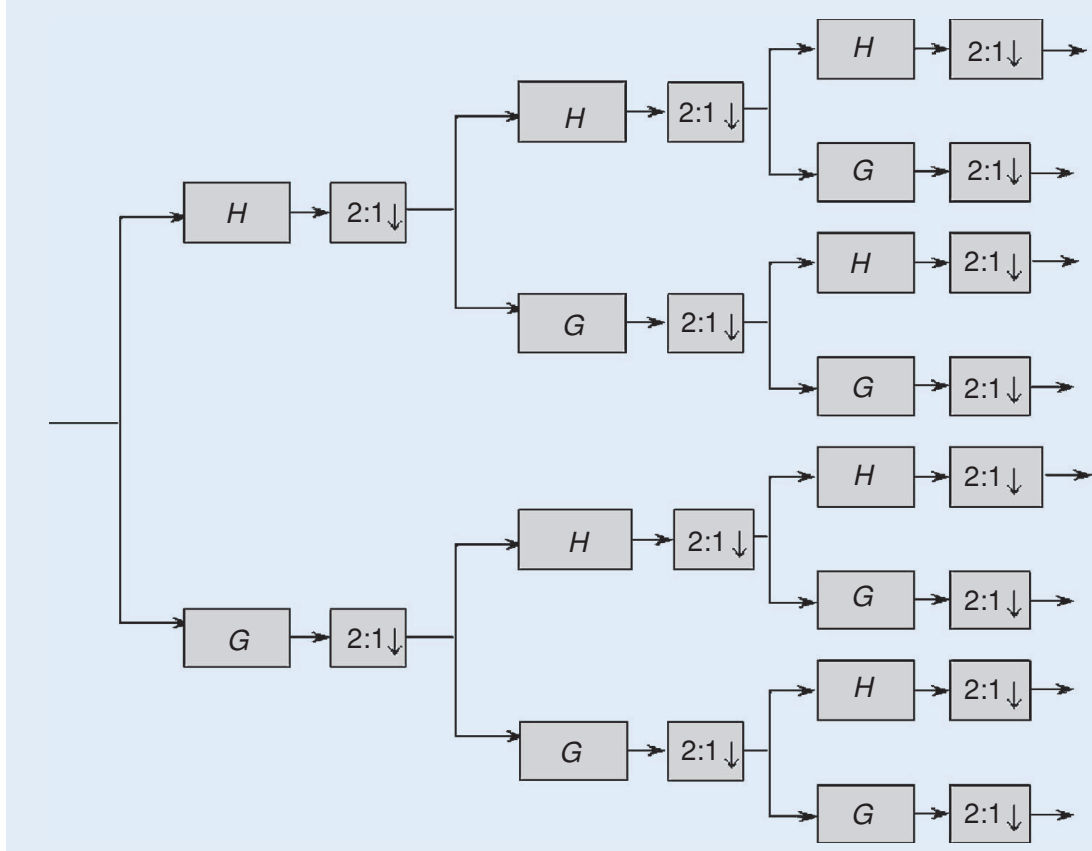

(b)

[FIG4] Generation of wavelets. (a) A level-3 tree gives eight wavelet packet bases for the transmitter and (b) their duals for the receiver. The up arrows represent interpolation by two, the down arrows represent decimation by two. $H^{\prime}$ and $G^{\prime}$ denote the frequency responses of the low and high pass reconstruction filters, while $H$ and $G$ denote the frequency responses of the low- and high-pass decomposition filters, respectively. 
smaller the transition bandwidth, the more frequency selective the filter will be.

Regularity corresponds to the smoothness and sidelobes of the signal. As the regularity order increases, so the sidelobes of the signal become lower and smoother. There will be trade off between $\mathrm{CR}$ performance and sidelobe reduction. The sidelobe can be depleted by increasing the regularity order with the cost of low filter frequency selectivity that will deteriorate the CR performance. More details on the analytical aspect of this subject can be found in [24]. The performance of MC-WPM with Remez exchange algorithm as CR system is evaluated in [25] and is compared with the Fourier-based MC-OFDM CR system in the presence of the LU signal in the additive white Gaussian noise (AWGN) channel. The results show that within certain Remez parameter values, MC-WPM outperforms the Fourierbased MC-OFDM.

\section{INTERPOLATED TREE ORTHOGONAL MULTIPLEXING (ITOM)}

In the MC-WPM technique, the filter banks perform the dual role of shaping the spectrum and also interpolating in time series. A more intelligent method is to separate the two processes in order to gain a greater control over the charac- teristics of the carriers. This method, called the ITOM method, was introduced in [13]. The spectral shaping is performed in a bank of shaping filters external to the wavelet packet tree structure. The procedure is depicted in Figure 5, where we can see that up-sampled shaping filters precede the input ports of the wavelet packet tree structure.

The prototype baseband spectral shape is preserved during the interpolation and aliasing operations. Notching over the desired spectral interval is achieved by vacating one or more of the input branches. In principal, the ITOM method will deliver the spectra formed by the input shaping filters to specific center frequencies by sequentially forming aliased spectral replicas by up sampling and selective half-band filtering the desired replica [13].

\section{SINGLE CARRIER CR SYSTEM}

Single-carrier transmission for opportunistic spectrum access has recently being initiated. Upon receiving the spectrum occupancy information, the time-domain signal can be shaped in a way that part of the spectrum will not distort the legacy system signal. Single carrier transmission using Fourier and wavelet transformation signal shaping techniques will be discussed will be discussed in the next sections.

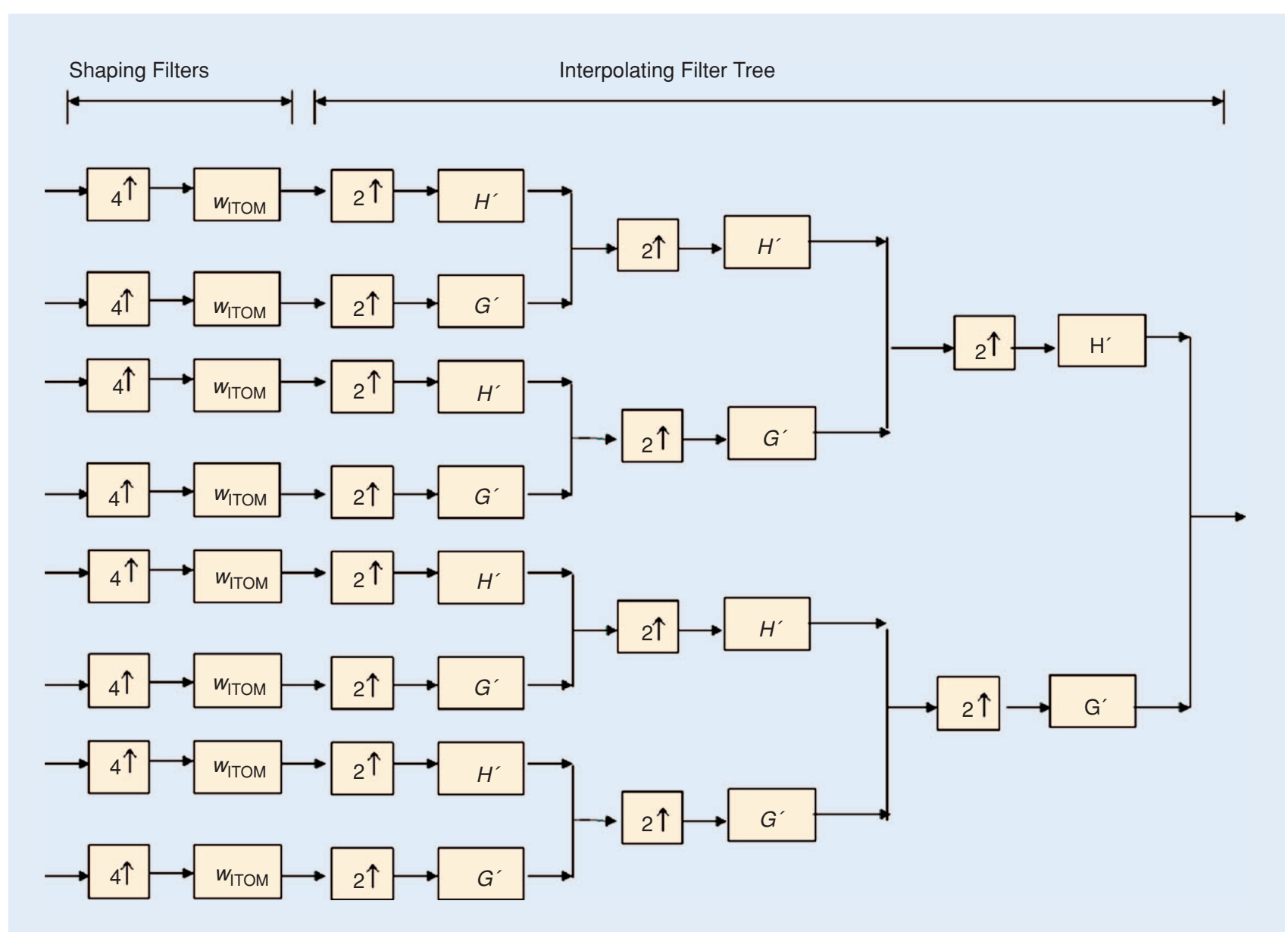

[FIG5] Modulator of ITOM interpolated tree structure [13] where $w_{\text {ITOM }}$ is the ITOM shaping filters. 


\section{TRANSFORM DOMAIN COMMUNICATIONS SYSTEM}

Transform domain communications system (TDCS) is a single carrier modulation that was initially introduced in [26]. TDCS fundamentally synthesizes a smart adaptive waveform to avoid interference at the transmitter, while the conventional technique tries to mitigate the interference at the receiver. Basically the spectral of interference signal or the LU's signal currently occupying the spectrum are scanned. Once they are indentified through some form of threshold detection, a clean spectrum information $A=\left\{A_{1}, A_{2}, \ldots, A_{\text {NFFT }}\right\}$ will be formed by the spectrum magnitude module, where amplitudes of interfering frequency components exceeding a threshold are set at zero, while the others are set at one. The multivalued complex pseudorandom phase vector $\left(e^{j \theta}\right)$ is multiplied element by element with $A$ to produce the spectral vector $B$. After IFFT operation, a time-domain basis function $b(t)$ is derived, which is subsequently stored and modulated by using pulse amplitude modulation (PAM), pulse position modulation (PPM), or cyclic code shift keying (CCSK). In CCSK, the bits are represented by the cyclic shifted pulse in time.

This zeros insertion impact can be described from the TDCS power spectral density (PSD)

$$
\operatorname{PSD}(f)=\left|\frac{1}{N_{\mathrm{FFT}}} \sum_{m=0}^{N_{\mathrm{FFT}-1}} A_{m} e^{-j 2 \theta_{m}} \int_{-(1+\alpha) \frac{T_{u}}{2}}^{(1+\alpha) \frac{T_{u}}{2}} p(t) e^{-j 2 \pi\left(f-f_{m}\right)^{t} d t}\right|^{2}
$$

where $T_{u}$ is the useful signal duration, $p(t)$ is the window function, $\alpha$ is the roll off factor of the window, and $\theta_{m}$ the phase on subband $m$ produced by the random phase module. The random phase can be derived from one of the $2^{r}$ phase shifts which are evenly spaced around a unit circle, while $r$ is the number of bits representing a complex point if a linear feedback shift registers is used to choose it. The primitive polynomial of the linear feedback shift registers can be configured for multiple access features so that each access has its own random phase sequence. The random phase generator should be designed in such a way that the resulting time domain basis function $b(t)$ has correlation properties similar to those of sampled noise. If $p(t)$ is a rectangular window and $\alpha$ is zero, the area within the integral can be replaced by $T_{u} \operatorname{sinc}\left(\left(f-f_{m}\right) T_{u}\right)$. Zero amplitude at the carrier $m$ will make the PSD on that carrier position zero, and due to the orthogonality among carriers the power contributions from the other carriers are also zero. In this way the TDCS can be used as CR modulation technique in overlay opportunistic spectrum access, since part of its spectrum on the LU band will not significantly impact the LU transmission. Equation (6) is almost similar to (1), only the power pm and symbol amplitude $S_{m}$ in (6) are replaced by the spectrum information $A_{m}$ and the random phase $\theta_{m}$.

TDCS gives a greater degree of freedom in choosing the window $p(t)$ to lower the sidelobes of its spectrum. Unlike OFDM, the spectrum sidelobes of the window for TDCS do not have to be zero on the frequency spacing interval (in OFDM this is described as the orthogonality between carriers), as long as its sidelobes are very small. Since data detection at the receiver is performed by correlating the received signal with the reference signal, the designed nonorthogonal spectrum e.g., by applying half sine window or Gaussian window to the transmitted signal will not affect data detection. Decorrelation for symbol demapping is simplified in [27] by choosing the index (position) of the maximum of absolute value of inverse Fourier transform of the product of the conjugate received signal and reference signal in frequency domain.

Recently, the modified the TDCS model is proposed by adding another bits source as a form of embedded symbol and by multiplying the frequency domain TDCS basis function with the embedded symbol [28]. The purpose is to increase the TDCS bit rate. This extra bits source can also be used to transmit CR side information. Assigning extra bits to the embedded symbol denotes allocating extra power to the TDCS basis function (increased SNR per symbol) that will lead to the data detection enhancement of the legacy data source (from PAM, PPM, or CCSK detection). The TDCS with extra embedded symbol transmitter and receiver schemes are depicted in Figure 6.

Due to the extra embedded symbol, the TDCS PSD would become

$$
\begin{aligned}
\operatorname{PSD}(f)= & \mid \frac{1}{N_{\mathrm{FFT}}} S e^{j \theta} \\
& \sum_{m=0}^{N_{\mathrm{FFT}-1}} A_{m} e^{-j \theta_{m}} \int_{-(1+\alpha) \frac{T_{u}}{2}}^{(1+\alpha) \frac{T_{u}}{2}} p(t) e^{-j 2 \pi\left(f-f_{m}\right)^{t}} d t \mid
\end{aligned}
$$

where $S$ and $\varphi$ are the embedded symbol magnitude and phase. In order not to alter the PSD amplitude, the value of $S$ should be one, therefore PSK is considered as the suitable embedded symbol modulation mode.

\section{WAVELET DOMAIN COMMUNICATIONS SYSTEM}

Another family of TDCS is the wavelet domain communications system (WDCS) [29], [30] where the Fourier transform operation in TDCS is replaced by wavelet transformation. The wavelet 
transform is used in this scheme to identify and establish an interference-free spectrum. It was initially performed to overcome the deficiencies of the TDCS in estimating the characteristics of non stationary interference, and the inefficiency of the Fourier-based spectral estimator causing spreading of the interference energy into adjacent spectral regions thus resulting in performance degradation [29]. In [30] the traditional wavelet transform is extended by introducing the wavelet packet decomposition technique to WDCS with the intention of increasing the transform adaptation over a larger class of interfering signals, to obtain finer high-frequency resolution, and more accurate electromagnetic spectral estimates. Through the use of adaptive thresholds and notches, subbands in the packet-based WDCS transform that contain the interference are effectively cancelled.

A WDCS time domain basis function $b(t)$ is obtained by performing an inverse wavelet transfom of the multiplication (point to point) between the spectrum occupancy vector $A$ and the random phase $e^{j \theta}$. The wavelet basis functions which are earlier applied in MC-WPM such as Coifflets, Daubechies, Haar,

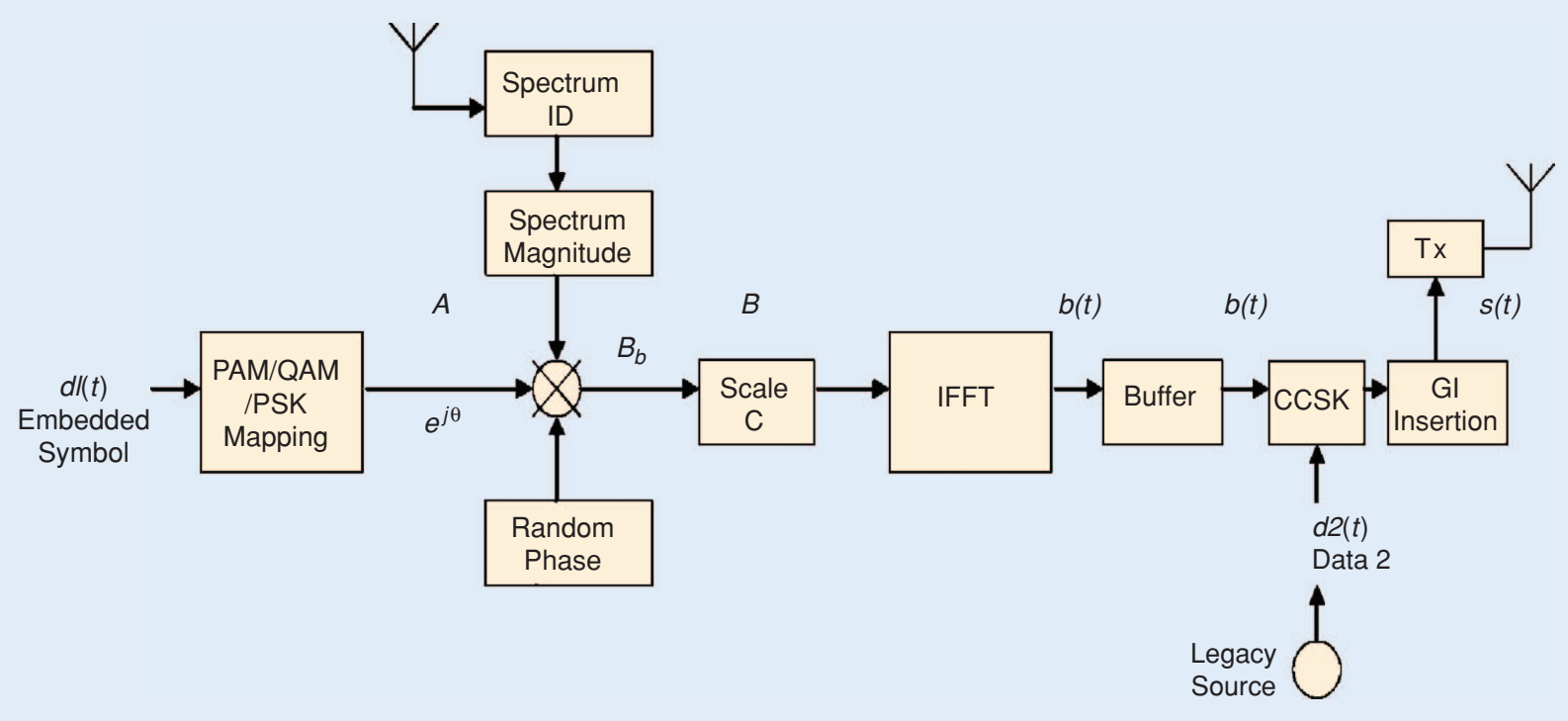

(a)

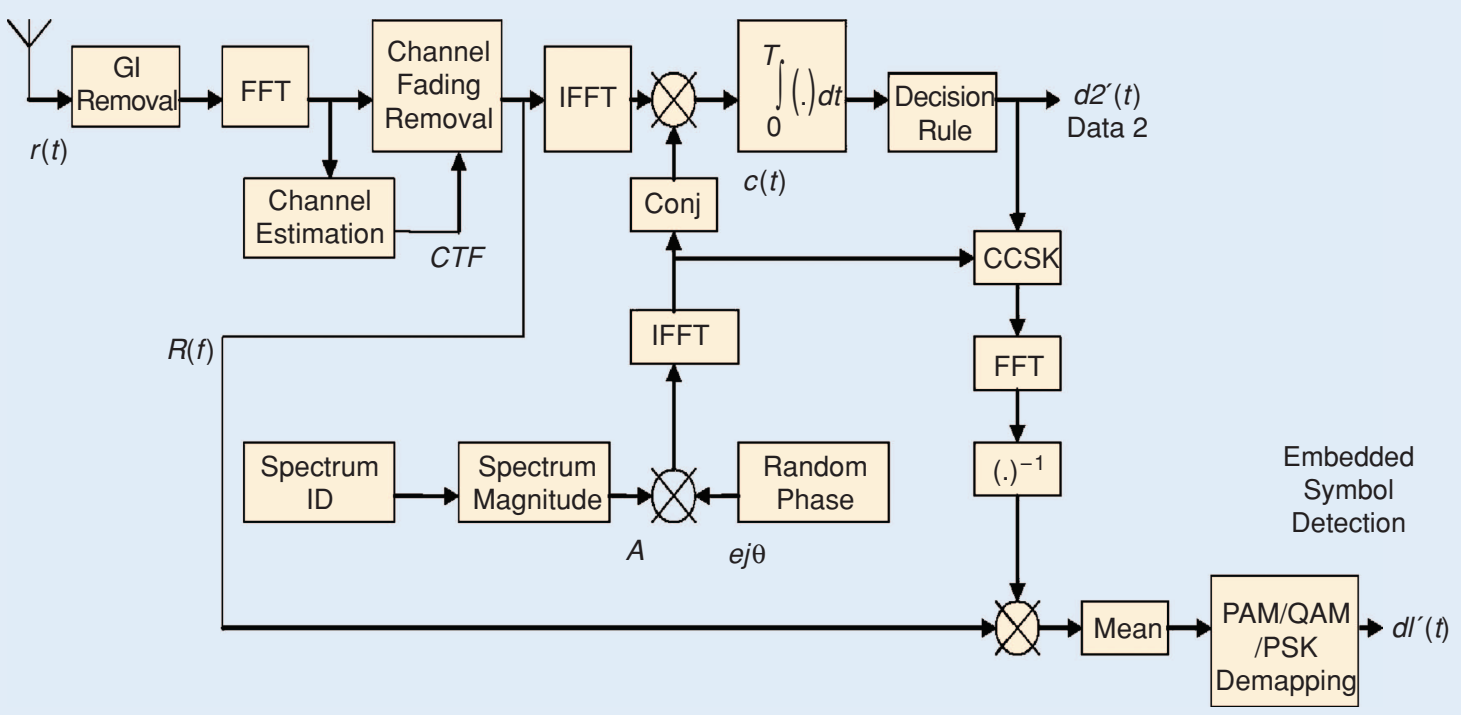

(b)

[FIG6] (a) Transmitter and (b) receiver architecture of TDCS with extra embedded symbol featuring channel estimation module. 
Symlets, or Remez filter can also be used in WDCS. The time domain basis function $b(t)$ is later modulated with the data using PAM, PPM, or CCSK modulation and then transmitted. The WDCS transmission block is presented in Figure 7.

At the receiver the detection is preceded by correlating the received signal with the reference signal in time domain. The receiver structure of WDCS is virtually the same as the TDCS, only the FFT and IFFT modules are replaced by respective discrete wavelet transform (DWT) and inverse discrete wavelet transform (IDWT).

\section{SUMMARY}

By relying on the accurate information from the spectrum sensing module about spectrum occupancy, a CR system can shape its signal in such a way that it coexists with the neighboring LUs and tries to achieve reliable communications while accessing the spectrum opportunistically. Of the several modulation techniques, it is the OFDM that is considered to be a strong candidate due to its spectrum access flexibility by notching its carriers on the position of LU's band. OFDM's main drawback is that its high sidelobes will interfere with the neighboring LU's band. Techniques such as windowing, adjacent carriers deactivation, cancellation carriers, multiple choice sequence, and a combination of all these techniques as the solutions used to mitigate the sidelobes problem have been overviewed. While windowing technique is a known application for sidelobes suppression, these techniques discussed in this article are novel for the CR. Bit-rate loss due to windowing can be compensated by the application of adaptive bit loading, where more bits are transmitted on the carriers with good SNR and fewer bits are transmitted on the carriers with bad SNR, while the target rate still can be obtained. Moreover, the OFDM symbols can be overlapped with the deliberate design of overlap position that will prevent the inter symbol interference.

The Fourier transform in OFDM can be replaced by taking the wavelet packetbased transform as an alternative technique. References have shown that the wavelet packet-based technique provides lower spectrum sidelobes compared to the regular OFDM with rectangular window. Among several wavelet basis functions, it is the one which is derived from the Remez exchange algorithm that provides a higher degree of flexibility in shaping its signal spectrum. By configuring the filter length, transition bandwidth, and regularity order of the filter, the desired CR spectrum can be designed.

Single carrier modulation for a CR system, known as TDCS, has been studied. The technique requires the spectrum occupancy information and relies for its data detection on the correlation properties of the generated random sequence used to construct its waveform. By utilizing the spectrum occupancy information and the random sequence, the TDCS can shape its signal to share the spectrum with the LUs. The shaping is employed by notching its energy in the band occupied by the LU. The combination with windowing will even shape its spectrum better. Since the data detection at the receiver is applied by cross-correlating the received signal with the reference signal, subcarrier unorthogonality will not severely affect the TDCS data detection. This condition indicates a huge degree of freedom when it comes to choose the window shape to lower the TDCS spectrum sidelobes. The data bits are aided to the signal by PAM, PPM, or CCSK modulation. Like OFDM, TDCS also requires an IFFT module to create its time domain signal. TDCS possesses lower bit rate compared to OFDM. As an effort to increase the TDCS bit rate, we have introduced additional data source to the TDCS as a form of embedded symbol by multiplying the frequency domain TDCS basis function with the embedded symbol. As embedding extra symbol to the TDCS basis function refers to adding extra energy to the TDCS symbol, the cross-correlation property of the TDCS symbol will be enhanced. As the outcome, the data BER from the legacy bit source (PAM, PPM, or CCSK) will be improved.

The Fourier transform in TDCS can be replaced by the wavelet transform that invokes the WDCS as another single carrier modulation technique for CR system. The data detection in WDCS is implemented by correlating the received WDCS signal with the time domain reference signal.

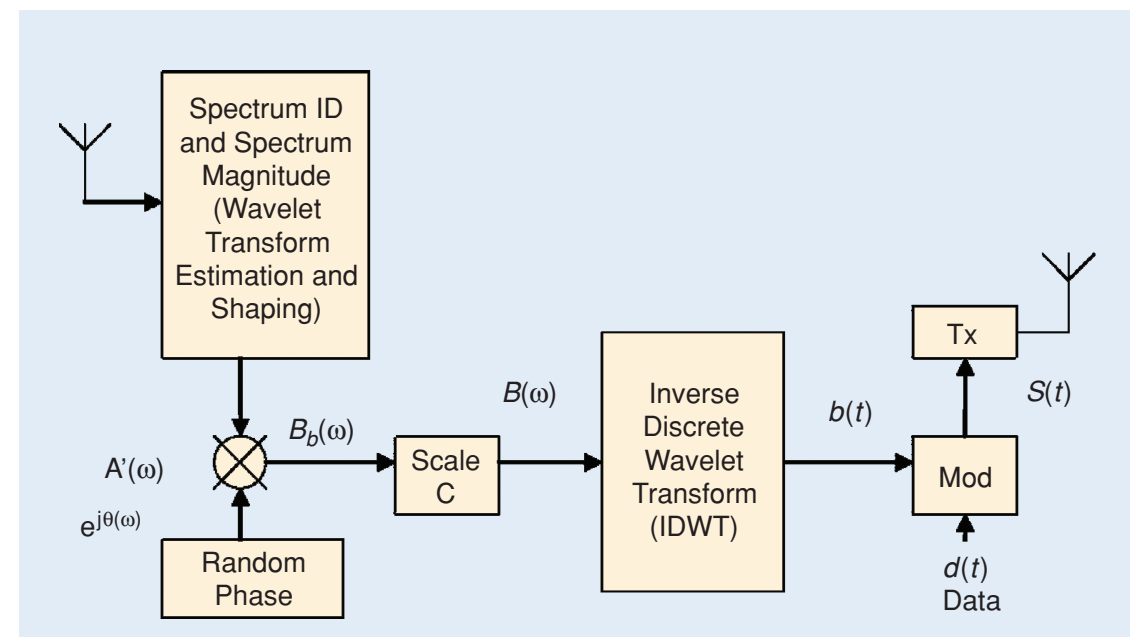

[FIG7] WDCS transmitter blocks [29]. 


\section{ACKNOWLEDGMENT}

The authors acknowledge the support of Dutch Adaptive Adhoc Freeband (AAF) project in this research.

\section{AUTHORS}

Ibrahim Budiarjo (i.budiarjo@ irctr.tudelft.nl) received his bachelor of engineering (Sarjana Teknik) degree in electronics from Bandung Institute of Technology, Indonesia, in 2001. He received his M.S. degree in communications engineering from Munich University of Technology (TU Muenchen), Germany, in 2003. He is working toward a Ph.D. degree in international research, at the Centre for Telecommunications and Radar, Delft University of Technology, The Netherlands. His research interests are adaptive signal processing for cognitive radio, including CDMA, OFDM, wavelet packet modulation, MIMO systems, beamforming, and embedded system for CR applications.

Homayoun Nikookar (h.nikookar@irctr.tudelft.nl) received his Ph.D. in electrical engineering from Delft University of Technology (TU Delft), The Netherlands, in 1995. $\mathrm{He}$ is an associate professor at the International Research Centre for Telecommunications and Radar (IRCTR) of the Department of Electrical Engineering, Mathematics and Computer Science of TU Delft. He is also the leader of the IRCTR Radio Advanced Technologies and Systems Program. He has conducted research in many aspects of wireless communications, including wireless channel modeling, UWB, MIMO, multicarrier transmission, wavelet-based OFDM, and CR. He is a Senior Member of the IEEE.

Leo P. Ligthart (1.p.ligthart@ irctr.tudelft.nl) received an engineering degree (cum laude) and a doctor of technology degree from Delft University of Technology (TU Delft) in 1969 and 1985, respectively. He received doctorates (honoris causa) at Moscow State Technical University of Civil Aviation in 1999 and Tomsk State University of Control Systems and Radioelectronics in 2001. In 1994, he became director of the International Research Center for Telecommunications and Radar. His research interests include antennas and propagation, radar, and remote sensing, but he has also been active in satellite, mobile, and radio communications. He is a Fellow of the IEEE.

\section{REFERENCES}

[1] S. Haykin, "Cognitive radio: Brain empowered wireless communications," IEEE J. Select. Areas Commun., vol. 23, no. 2, pp. 201-220, Feb. 2005.

[2] J. Mitola III, "Cognitive radio an integrated agent architecture for software defined radio," Ph.D. dissertation, Dept. Teleinformatics, KTH Royal Institute of Technology, Stockholm, Sweden, 2000.

[3] R.W. Brodersen, A. Wolisz, D. Cabric, S.M. Mishra, and D. Wilkomm, CORVUS: A Cognitive Radio Approach for Usage of Virtual Unlicensed Spectrum. White Paper, Dept of Elect. Eng. and Comp. Sci., Univ. California, Berkeley, July, 2004 [Online]. Available: http://bwrc.eecs.berkeley.edu/Research/MCMA/ CR_White_paper_final1.pdf

[4] T. Weiss, J. Hillenbrand, A. Krohn, and F.K. Jondral, "Mutual interference in OFDM-based spectrum pooling systems," in Proc. IEEE Vehicular Technology Conf., Milan, Italy, 17-19 May 2004, pp. 1873-1877.

[5] T. Weiss and F. Jondral, "Spectrum pooling-An innovative strategy for the enhancement of spectrum efficiency," IEEE Commun. Mag., vol. 42, no. 3, pp. S8--14, Mar. 2004.

[6] H. Nikookar and R. Prasad. "Wave shaping of multicarrier signal for data transmission over wireless channels," in Proc. 6th IEEE Int. Conf. Universal Personal Communications Record (ICUPC), 1997, pp. 173-177.
[7] P. Tan and N.C. Beaulieu. "Reduced ICI in OFDM systems using the better than raised-Cosine pulse," IEEE Commun. Lett., vol. 8, no. 3, pp. 135-137, Mar. 2004.

[8] A. Assalini and A.M. Tonello, "Improved nyquist pulses," IEEE Commun. Lett., vol. 8 , no. 2 , pp. 87-89, 2004.

[9] C. Muschalik, "Improving an OFDM reception using an adaptive nyquist windowing," IEEE Trans. Consumer Electron., vol. 42, no. 3, pp. 259-269, 1996.

[10] K. Witrisal, "OFDM air interface design for multimedia communications," Ph.D. dissertation, Dept. Elect. Eng., Math. and Comp. Sci., Delft Univ. Technology, The Netherlands, Apr. 2002.

[11] I. Budiarjo, H. Nikookar, and L.P. Ligthart, "Combined spectrum pooling and adaptive bit loading for cognitive radio OFDM based aystem," in Proc. 13th IEEE SCVT, Liege, Belgium, Nov. 2006, pp. 73-76.

[12] H. Nikookar and R. Prasad, "Optimal waveform design for multicarrier transmission over a multipath channel," in Proc. 47th IEEE Vehicular Technology Conf., Phoenix, AZ, May 4-7 1997, pp. 1812-1816.

[13] F. Harris and E. Kjeldsen, "A novel interpolated tree orthogonal multiplexing (ITOM) dcheme with compact time-frequency localization: An introduction and comparison to wavelet filter banks and polyphase filter banks," in Proc. Software Defined Radio Technical Conf. and Product Exposition, Orlando, FL, Nov. 2006.

[14] S. Brandes, I. Cosovic, and M. Schnell, "Reduction of out-of-band radiation in OFDM based overlay systems," in Proc. 1st IEEE Int. Symp. New Frontiers in Dynamic Spectrum Access Networks (DySPAN), Baltimore, MD, Nov. 2005, pp. 662-665.

[15] I. Cosovic, S. Brandes, and M. Schnell, "Subcarrier weighting: A method for sidelobe suppression in OFDM systems," IEEE Commun. Lett., vol. 10, no. 6, pp. 444-446, June 2006.

[16] H. Nikookar and R. Prasad, "Weighted OFDM for wireless multipath channels," IEICE Trans. Commun., vol. E83-B, no. 8, pp. 1864-1872, Aug. 2000.

[17] I. Cosovic and T. Mazzoni, "Suppression of sidelobes in OFDM systems by multiple choice sequences," European Transactions on Telecommunications (Special Issue on Multi Carrier-Spread Spectrum,) pp. 623-630, June 2006.

[18] H.A. Mahmoud and H. Arslan, "Sidelobe suppression in OFDM-based spectrum sharing systems by using adaptive symbol transition," IEEE Commun. Lett., vol. 12 , no. 2, pp. 133-135, Feb. 2008.

[19] M. Vetterli and I. Kovacevic, Wavelets and Subband Coding. Englewood Cliffs, NJ: Prentice-Hall, 1995.

[20] A. Jamin and P. Mahonen, "Wavelet packet modulation for wireless communications," Wireless Commun. Mobile Comput. J., vol. 5, no. 2, pp. 123-137, Mar. 2005.

[21] C.V. Bouwel, J. Potemans, S. Schepers, B. Nauwelaers and A.V. Capelle, "Wavelet packet based multicarrier modulation," in Proc. IEEE Benelux Symp. Communications and Vehicular Technology, Leuven, Belgium, Oct. 2000, pp. $131-138$.

[22] B.G. Negash, and H. Nikookar, "Wavelet based multicarrier transmission over multipath wireless channels," IEE Electron. Lett., vol. 36, no. 21, pp. 1787-1788, Oct. 2000.

[23] Y. Zhang and S. Cheng, "A novel multicarrier signal transmission system over multi-path channel of low-voltage power line," IEEE Trans. Power Delivery, vol. 19, no. 4, pp. 1668-1672, Oct. 2004.

[24] O. Rioul and P. Duhamel, "A Remez exchange algorithm for orthonormal wavelets," IEEE Trans. Circuits Syst. II, vol. 41, no. 8, pp. 550-560, Aug. 1994.

[25] M.K. Lakshmanan, I. Budiarjo, and H. Nikookar, "Maximally frequency selective wavelet packets based multi-carrier modulation scheme for cognitive radio systems," in Proc. 50th IEEE Global Communications Conf. (Globecom), Washington, D.C., Nov. 2007, pp. 4185-4189.

[26] V. Chakravarthy, A.S. Nunez, J.P. Stephens, A.K. Shaw, and M.A. Temple, "TDCS, OFDM, and MC-CDMA: A brief tutorial," IEEE Commun. Mag., vol. 43, no. 9, pp. S11-S16, Sept. 2005.

[27] G.M. Dillard, M. Reuter, J. Zeiddler, and B. Zeidler, "Cyclic code shift keying: A low probability of intercept communication technique," IEEE Trans. Aerosp. Electron. Syst., vol. 39, no. 3, pp. 786-798, July 2003.

[28] I. Budiarjo, H. Nikookar, and L.P. Ligthart, "On the utilization of embedded symbol for CCSK BER improvement in TDCS dynamic spectrum access," to appear in Proc. IEEE European Wireless Technology Conf., 28-29 Oct. 2008, Amsterdam, The Netherlands.

[29] M.J. Lee, M.A. Temple, R.L. Claypoole, Jr., R.A. Raines, and J.P. Stephens, "Wavelet domain communication system: Bit error sensitivity characterization for geographically separated transceivers," in Proc. MILCOM, Anaheim, CA, Oct. 2002, vol. 2, pp. 1378-1382.

[30] M.J. Lee, "Wavelet domain communication system (WDCS): Packet-based wavelet spectral estimation and M-ARY signaling," M.S. thesis, AFIT/GE/ENG/02M-14, Grad. School Eng. and Management Air Force Inst. Tech., Wright-Patterson Air Force Base, OH, DTIC:ADA401433, Mar. 2001. 